\title{
Intergenerationale und professionelle Unterstützung älterer Personen in Europa
}

In Europa erhöht sich der Anteil älterer Menschen stetig. In Zukunft wird daher einerseits der Hilfe- und Pflegebedarf ansteigen, während andererseits immer weniger Personen zur Verfügung stehen, die diese Unterstützung leisten können. Vielen droht eine Unterversorgung mit sozial-medizinischen Leistungen. Die Suche nach möglichen Lösungswegen lenkt den Blick auf die vielfältigen Hilfe- und Pflegearrangements, die es andernorts gibt: Wie ist Unterstützung älterer Menschen in europäischen Ländern organisiert und wer leistet sie? Wie sind die Altersrisiken einer Unterversorgung an Hilfe- und Pflegeleistungen in der Bevölkerung verteilt?

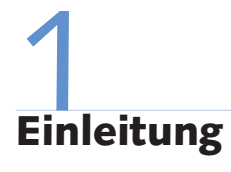

In Politik und Gesellschaft werden die Auswirkungen der demografischen Alterung intensiv diskutiert. Dabei interessiert vor allem die familiale und institutionelle Absicherung der altersbedingten Risiken, jeweils mit dem Blick auf drohende Finanzierungslücken in den sozialen Sicherungssystemen. In der Wissenschaft, insbesondere in der Soziologie, rücken ältere Menschen ebenfalls in den Fokus. Zwar wird hier immer häufiger das Potenzial der älteren Menschen thematisiert, zum Beispiel die große Bedeutung der Großeltern für die Enkelkinder(-betreuung) (Hank/ Buber 2009; Höpflinger et al. 2006; Igel et al. 2009). Dennoch steht auch hier die Frage im Raum, wie der altersbedingte Bedarf an Hilfe- und Pflegeleistungen gedeckt werden kann, wenn einerseits der Anteil älterer Menschen stark ansteigt und andererseits immer weniger jüngere Menschen die Unterstützung übernehmen können oder wollen (Blinkert/Klie 2004; BMFSFJ 2006, S. 142f.).

Auch in der Familie ist die Pflege und Hilfe älterer Angehöriger ein präsentes und bedeutendes Thema. Wer kann oder sollte die Unterstützung übernehmen? Ist die Hilfe und Pflege der Eltern mit dem Familien- und Berufsleben vereinbar? Welche weiteren Möglichkeiten zur Unterstützung der älteren Angehörigen gibt es? Können professionelle Hilfe- und Pflegeleistungen in Anspruch genommen werden? Bei der Wahl des Hilfe- und Pflegearrangements handelt sich um eine komplexe Entscheidung, die von vielfältigen Faktoren abhängt. Neben individuellen und familialen
Eigenschaften wie zum Beispiel Gesundheitszustand, Einkommen, Erwerbsstatus, Wohnentfernung und Familienzusammensetzung sind dabei auch kulturelle Normen und wohlfahrtsstaatliche Leistungen sowie deren Zusammenspiel von Bedeutung (Haberkern 2009; Lowenstein/ Ogg 2003; Pfau-Effinger 2005).

In den folgenden Abschnitten wird die familiale und gesellschaftliche Organisation der Hilfe und Pflege älterer Menschen beleuchtet. ${ }^{1}$ Dabei wird zunächst der Hilfe- und Pflegebedarf in Privathaushalten in sieben europäischen Ländern (Belgien, Dänemark, Deutschland, Italien, Niederlande, Österreich, Schweden) beschrieben (Abschnitt 2). Danach wird den Fragen nachgegangen, ob und wie dieser Bedarf gedeckt wird (Abschnitt 3). Wie ist die Unterstützung älterer Menschen organisiert? Welche Rolle spielt die Familie, welche kommt professionellen hauswirtschaftlichen und sozialmedizinischen Diensten zu? Welche Unterschiede und Gemeinsamkeiten gibt es zwischen europäischen Ländern? Von besonderem Interesse ist dabei eine gemeinsame Betrachtung von informellen und professionellen Dienstleistungen bzw. deren Ineinandergreifen (Abschnitt 4; Attias-Donfut/Wolff 2000; Daatland/ Herlofson 2003; Motel-Klingebiel/TeschRömer 2006). Unterschiede zwischen den Ländern können dabei wesentlich auf Unterschiede in der informellen Unterstützung durch Angehörige und Bekannte zurückgeführt werden. Kinder übernehmen häufig die Hilfe- und Pflegeleistungen, weshalb wir im Folgenden vor allem intergenerationale Unterstützungsleistungen in den Blick nehmen.

Schließlich wird die familiale Entscheidung für Hilfe- und Pflegearrangements untersucht, wobei wir zwischen einem in- tergenerationalen, einem professionellen und einem kombinierten Unterstützungsarrangement unterscheiden. Beim intergenerationalen Hilfe- und Pflegearrangement erfolgt die informelle Unterstützung durch die Kinder, beim professionellen durch sozial-medizinische Fachkräfte, während im kombinierten Unterstützungsarrangement die familiale und professionelle Unterstützung gleichzeitig erfolgen und damit vermutlich auch ineinander greifen (Brandt 2009, S. 103ff.; Haberkern 2009, S. 134ff.).

\footnotetext{
1 In den empirischen Analysen verwenden wir Daten aus dem Release 2.0.1 der SHARE-Daten. Detaillierte Informationen über das Projekt finden sich unter www.share-project.org, zu methodischen Details siehe Börsch-Supan/Jürges 2005 Die SHARE-Datenerhebung wurde hauptsächlich durch das 5. und 6. Forschungsrahmenprogramm der Europäischen Union finanziert (Projekte QLK6-CT-2001-00360; RII-CT-2006-062193; CIT5-CT-2005-028857). Weitere Finanzmittel wurden vom US National Institute on Aging (U01 AG09740-13S2, P01 AG005842, P01 AG08291, P30 AG12815, Y1-AG-4553-01, OGHA 04-064, R21 AG025169) sowie nationalen Geldgebern zur Verfügung gestellt.
}

Klaus Haberkern, Dr., Soziologe, arbeitet als wissenschaftlicher Assistent am Soziologischen Institut der Universität Zürich und in der Forschungsgruppe AGES. Arbeitsschwerpunkte: Familie, Arbeit, Wohlfahrtsstaat, Zeitverwendung. e-mail: haberkern@soziologie.uzh.ch Martina Brandt, Dr., Soziologin, ist wissenschaftliche Mitarbeiterin am Mannheim Research Institute for the Economics of Aging (MEA) im Forschungsbereich Survey of Health, Aging and Retirement in Europe (SHARE) Arbeitsschwerpunkte: Soziale Netzwerke, Arbeit, Gesundheit. e-mail: brandt@mea.uni-mannheim.de 

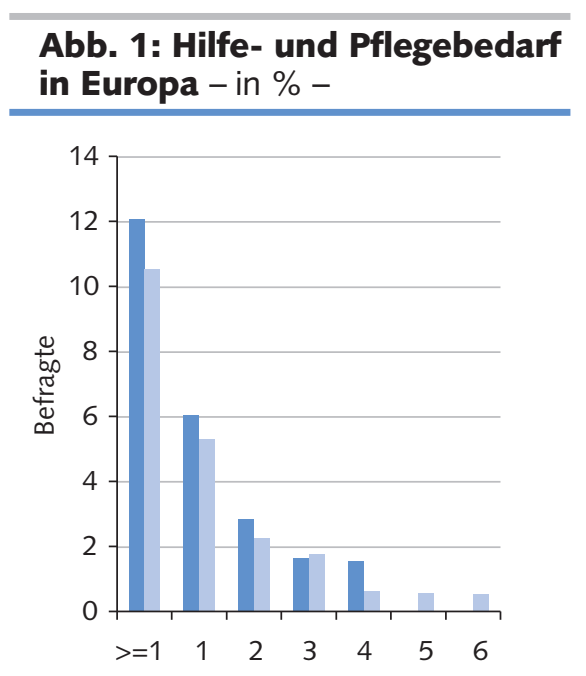

Anzahl der Einschränkungen (IADL/ADL) - Hilfe Pflege

Quelle: Darstellungs der Autoren; $\mathrm{n}=18.186$ Personen; SHARE 2004 release 1.2

WSI MITTEILUNGEN

Dabei stehen folgende Fragen im Mittelpunkt: Welche Faktoren begünstigen die Pflege durch Kinder? Warum werden manche betagten Eltern von ihrem Nachwuchs unterstützt, andere jedoch von professionellen Pflegekräften und Dienstleistern? Welche Faktoren begünstigen eine Kooperation zwischen Angehörigen und Fachkräften? Im Fazit (Abschnitt 5) fassen wir die Befunde zusammen und diskutieren sie als Herausforderung für die gesellschaftliche Organisation der Unterstützung älterer Menschen insbesondere in Deutschland, Österreich und Italien.

\section{Hilfe- und Pflegebedarf in Europa}

Um die gesellschaftliche Organisation der Hilfe und Pflege älterer Menschen untersuchen und bewerten zu können, müssen zuerst für den Vergleich geeignete Indikatoren bestimmt werden. Neben der Organisation und der Aufgabenteilung zwischen informellen Helfern und Pflegepersonen einerseits und Fachkräften andererseits interessiert die Leistungsfähigkeit der Unterstützungssysteme, also ob die Unterstützung dem Bedarf angepasst ist und inwieweit der Hilfe- und der Pflegebedarf in einer Gesellschaft gedeckt werden. Zunächst bestimmen wir die beiden Unterstützungsfor-
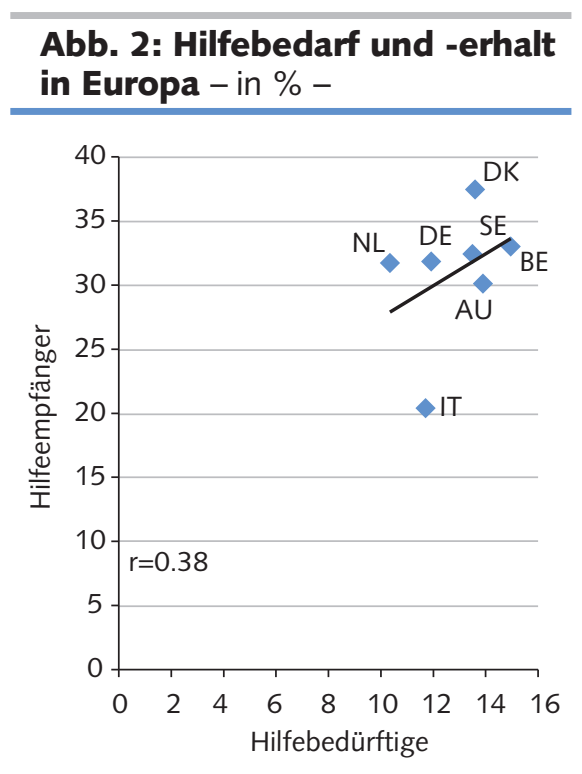

Quelle: Berechnungen der Autoren, gewichtet, $\mathrm{n}=7$ Länder; SHARE 2004 release 1.2 .

\section{WSI MITTEILUNGEN}

men Hilfe und Pflege näher und ermitteln den Hilfe- und Pflegebedarf in den Ländern.

Hilfe und Pflege unterscheiden sich in verschiedenen Aspekten. Pflege ist sehr zeitintensiv und erfolgt meist regelmäßig. Hilfeleistungen sind hingegen nicht unbedingt an einen (längerfristigen) Bedarfgekoppelt. Während auf Pflegeleistungen demnach häufig nicht verzichtet werden kann, stellen Hilfen oft auch eine willkommene, jedoch nicht zwingend notwendige Erleichterung dar. Familiale Hilfe- und Pflegeleistungen hängen deshalb von unterschiedlichen Faktoren ab. Im Folgenden unterscheiden wir zwischen Hilfebedarf und -leistungen einerseits und Pflegebedarf und -leistungen andererseits. Hilfebedarf und Hilfeleistungen bezeichnen dabei eine Unterstützung bei alltäglichen Aufgaben (instrumental activities of daily living (IADL) wie beispielsweise Haus-, Reparatur- und Gartenarbeiten); Pflegebedarf und Pflegeleistungen umfassen die Unterstützung bei basalen Tätigkeiten (activities of daily living (ADL) wie Aufstehen/Hinlegen, Anziehen, Gehen, Essen, Waschen, Toilettengang (vgl. Walker et al. 1995).

Wie in Abbildung 1 dargestellt, benötigen $12 \%$ der Befragten ab 50 Jahren Hilfe bei mindestens einer alltäglichen Aufgabe (Zubereitung einer warmen Mahlzeit, Einkaufen von Lebensmitteln, Arbeiten im Haus oder im Garten, mit Geld umgehen, zum Beispiel Rechnungen bezahlen oder Ausgaben kontrollieren). Davon sind etwa
$6 \%$ nur bei einer der genannten Erledigungen hilfebedürftig und $3 \%$ nennen zwei Einschränkungen. Jeweils weniger als $2 \%$ der Befragten benötigen bei drei oder vier alltäglichen Aktivitäten Unterstützung.

Etwa ein Zehntel (und damit weniger ältere Personen) sind pflegebedürftig bzw. benötigen Unterstützung beim Anziehen, quer durch einen Raum gehen, Baden oder Duschen, Essen, sich ins Bett legen, aus dem Bett aufstehen und/oder Benutzen der Toilette (Abbildung 1). Hier sind es $5 \%$, die angeben, bei einer dieser Aktivitäten Unterstützung zu benötigen, je etwa $2 \%$ nennen drei und vier alltägliche Aufgaben, die sie nicht mehr allein verrichten können. Vier, fünf und sechs Einschränkungen geben weniger als $1 \%$ der Befragten an.

Die Befunde zeigen also, dass die meisten Personen in Privathaushalten nur vergleichsweise wenige Einschränkungen gleichzeitig hinnehmen müssen: Ein mehrfacher, sehr intensiver Hilfe- und Pflegebedarf ist nur sehr selten anzutreffen, u.a., da diese Personen vermehrt in stationären Einrichtungen untergebracht werden. Da die Erhebung in den meisten Ländern Personen in Kollektivhaushalten wie Alters- und Pflegeheimen nicht umfasst, kann der Personenkreis in sozialmedizinischen Einrichtungen hier nicht berücksichtigt werden.

\section{3 Familiale und gesellschaft-
liche Organisation von
Hilfe und Pflege}

Sobald der Hilfe- oder Pflegebedarf bekannt ist, stellt sich zwangsläufig die Frage, ob und inwieweit dieser auch gedeckt werden kann. Können hilfe- und pflegebedürftige Personen in Europa auf Unterstützung zählen?

Stellt man dem gesamtgesellschaftlichen Bedarf den Empfang von Hilfe- und Pflegeleistungen gegenüber (Abbildung 2 und Abbildung 3), zeigt sich deutlich, dass mit steigendem Hilfe- und Pflegebedarf in den sieben untersuchten Ländern auch die empfangenen Leistungen zunehmen.

Dabei ist besonders interessant, dass der Anteil an Hilfeempfängern höher ist als der Anteil der Hilfebedürftigen. Hilfeleistungen werden demnach auch geleistet, wenn sie nicht unbedingt benötigt werden. Hilfe bei IADL kann damit zwar einem länger- 


\section{Abb. 3: Plegebedarf und -erhalt in Europa - in \% -}

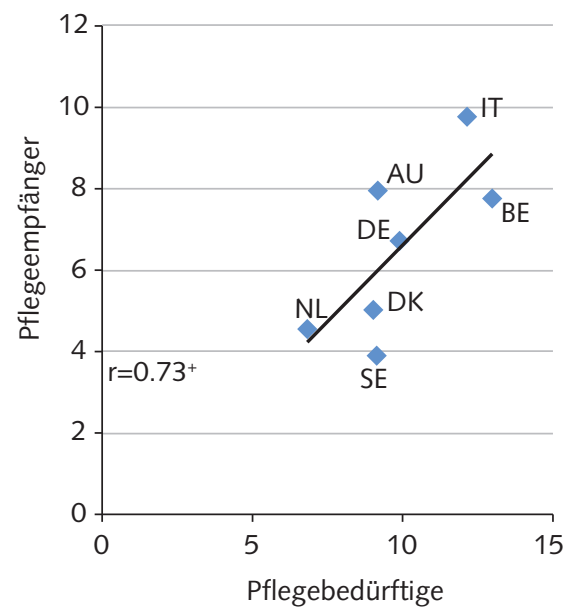

Quelle: Darstellung der Autoren; SHARE 2004 release 1.2; Berechnungen der Autoren, gewichtet. Korrelation signifikant auf dem ${ }^{+}=10 \%$-Niveau, $n=7$ Länder.

WSI

\section{Abb. 4: Hilfearrangements in Europa - in \% -}

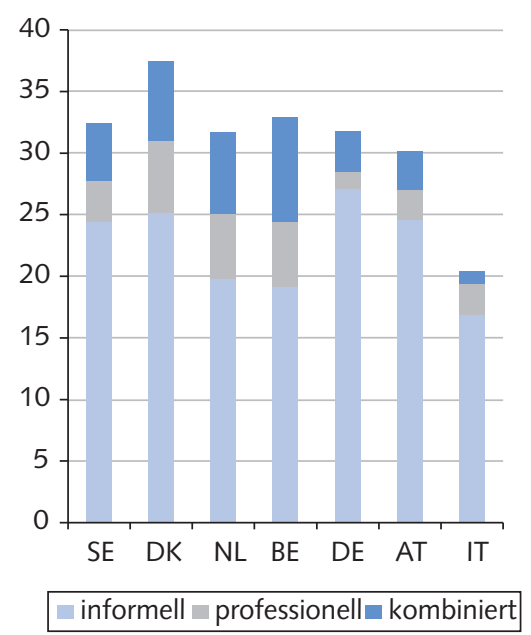

Quelle: Darstellung der Autoren; $\mathrm{n}=12.765$ Haushalte; SHARE 2004 release 1.2 .

WSI MITTEILUNGEN

\section{Abb. 5: Pflegearrangements in} Europa - in \% -

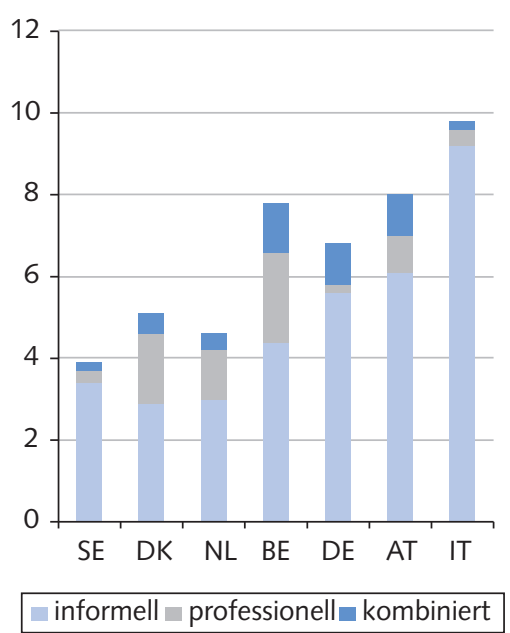

Quelle: Darstellung der Autoren; $\mathrm{n}=18.186$ Haushalte: SHARE 2004 release 1.2

\section{WSI MITTEILUNGEN}

fristigen Unterstützungsbedarf folgen, wird aber auch aus Dankbarkeit oder als Zuwendung geleistet, wenn kein aktueller Bedarf besteht. Der Zusammenhang zwischen Bedarf und Leistung ist damit weniger zwingend als bei Pflege, und entsprechend ist der statistische Zusammenhang zwischen Hilfebedarf und -erhalt in europäischen Gesellschaften nicht signifikant. Pflege wird hingegen - zumindest auf gesellschaftlicher Ebene-nur dann geleistet, wenn auch ein Pflegebedarf vorhanden ist, wobei es deutlich mehr Pflegebedürftige als -empfänger gibt (Abbildung 3). Mit steigendem Pflegebedarf in einer Gesellschaft erhalten auch mehr Personen Pflegeleistungen. Allerdings weist die pflegerische Versorgung im Unterschied zu Hilfeleistungen Lücken auf, denn in allen Ländern - und damit auch in den skandinavischen Wohlfahrtsstaaten - erhält ein beträchtlicher Anteil der Pflegebedürftigen keine Pflegeleistungen, mitunter ist deutlich mehr als ein Drittel von einer Unterversorgung betroffen. Insgesamt muss sogar von einer noch ausgeprägteren Unterversorgung ausgegangen werden, da nicht alle Pflegeempfänger auch so umfassende Leistungen erhalten wie sie benötigen (Schneekloth/Leven 2003, S. 32).

Bei der Organisation der Hilfe und Pflege lässt sich zwischen einem informellen, einem professionellen und einem kombinierten Hilfe- und Pflegearrangement unterscheiden. Im informellen Pflegearrangement erhält die ältere Person ausschließlich von Angehörigen, Freunden und Bekannten Unterstützung, während im professionellen Arrangement nur Fachkräfte die Unterstützung leisten. Im kombinierten Fall greifen die informelle und professionelle Unterstützung ineinander, z. B. wenn Angehörige und ambulante Pflegedienste die Pflege gemeinsam übernehmen.

Welchen Anteil der Unterstützung übernehmen Privatpersonen und welchen Beitrag leisten professionelle Hilfe- und Pflegekräfte in den europäischen Ländern? Abbildung 4 zeigt deutlich, dass das Gros der Hilfe informeller Art ist: In allen Ländern kommt die Hilfe in mehr als der Hälfte der Fälle ausschließlich von Angehörigen oder Bekannten, wobei es deutliche Unterschiede gibt. In den konservativen Wohlfahrtsstaaten und Italien, einem Vertreter des familialistischen Wohlfahrtsregimes, beträgt diese Quote zwischen $81 \%$ und $85 \%$. In den skandinavischen und den Benelux-Staaten, die mit Ausnahme von Belgien dem sozialdemokratischen Wohlfahrtsregime zugerechnet werden, sind es zwischen $57 \%$ und $75 \%$. Professionelle Fachkräfte leisten dagegen nur einen kleinen Teil der Hilfe und Pflege, wobei die Unterstützung häufig gleichzeitig von Privatpersonen und professionellen Fachkräften kommt.

Je nach Land erhalten damit zwischen $20 \%$ und $37 \%$ der Haushalte älterer Menschen Hilfeleistungen. In den sozialdemokratischen Wohlfahrtsstaaten erhalten tendenziell mehr Personen und Paare $^{2}$ eine Unterstützung bei Tätigkeiten im und rund um den Haushalt - nicht zuletzt aufgrund der größeren Verbreitung von professionellen Hilfen.

Der Vergleich mit Pflegeleistungen (Abbildung 5) zeigt Gemeinsamkeiten, aber auch beträchtliche Unterschiede. Wie bei der Hilfe erhält die Mehrzahl der Pflegeempfänger ausschließlich informelle Unterstützungsleistungen, wobei in den konservativen und familialistischen Wohlfahrtsstaaten die Angehörigen und Bekannten stärker in die Pflege involviert sind als in den sozialdemokratischen Regimen. Gleichzeitig und im Unterschied zur Hilfe erhalten in den sozialdemokratischen Ländern weniger als 5 \% der Personen in Privathaushalten pflegerische Unterstützung. In den konservativen und familialistischen Wohlfahrtsstaaten sind es zwischen $6 \%$ und $8 \%$.

Insgesamt sind Hilfeleistungen damit deutlich weiter verbreitet als Pflegeleistungen. Einerseits erfordern sie meist weniger Aufwand, andererseits ist nur eine Minderheit auf Pflegeleistungen zwingend angewiesen. Auch wenn Hilfeleistungen

2 Ambulante Haushaltshilfe erhält (im Gegensatz zur persönlichen Pflege) der gesamte Haushalt, weshalb die Analyse hier auf Haushaltsebene erfolgt. 


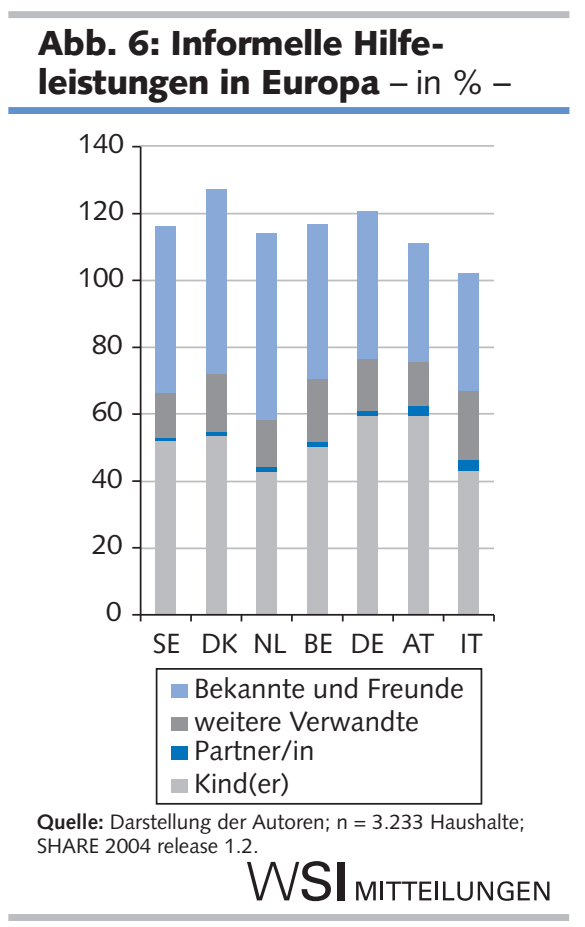

also weniger öffentliche Aufmerksamkeit erfahren als die (Angehörigen-)Pflege, darf ihre Bedeutung schon allein aufgrund ihres immensen gesamtgesellschaftlichen Umfangs keinesfalls unterschätzt werden.

Der Vergleich zwischen den Ländern zeigt, dass insbesondere das Ausmaß der informellen Unterstützung variiert. Länderunterschiede in der Unterstützung mit Hilfe- und Pflegeleistungen sind damit wesentlich auf Unterschiede in der informellen Unterstützung zurückzuführen. Im Folgenden werden diese daher genauer in den Blick genommen.

Abbildung 6 und Abbildung 7 stellen die Anteile der Hilfe- bzw. Pflegeempfänger dar, die informelle Unterstützung erhalten. Einige dieser Befragten werden von mehreren und verschiedenen Personen unterstützt, sodass sich die Anteile zu über $100 \%$ aufaddieren. Die Aufschlüsselung der Hilfeleistungen zeigt, dass ältere Menschen mehrheitlich von ihren Kindern unterstützt werden. Neben Kindern leisten auch Bekannte und Freunde häufig Hilfe, in den skandinavischen Ländern mehr als in den mittel- und südeuropäischen Staaten. Partnerinnen und Partner sind hier nahezu nicht vertreten, da nur haushaltsexterne Personen berücksichtigt wurden. ${ }^{3}$

Pflegeleistungen konnten dagegen auch innerhalb eines Haushalts erfasst werden. Entsprechend hoch ist hier auch der Anteil der Partnerpflege. Die meisten Empfänger

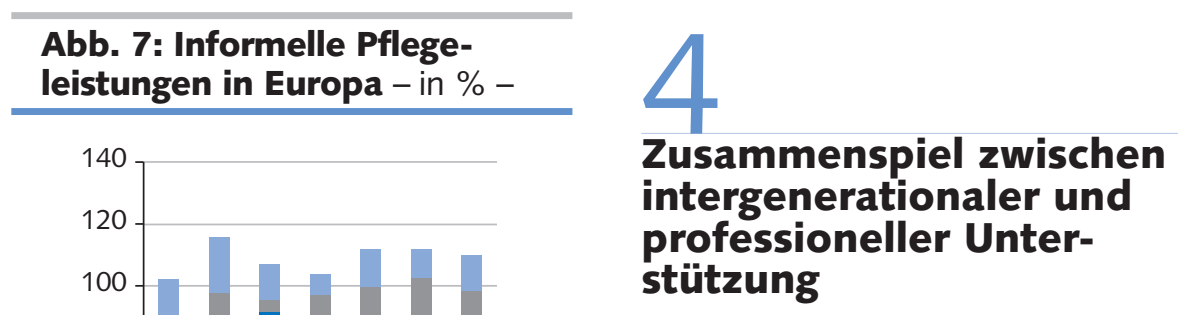

\subsection{HÄUFIGKEITEN}

Im Folgenden sollen nun die nationalen Hilfe- und Pflegesysteme verglichen werden, wobei die Verbreitung des intergenerationalen, des professionellen und kombinierten Unterstützungsarrangements als Indikatoren verwendet werden. Setzt man dabei intergenerationale und professionelle Unterstützungsleistungen in den einzelnen Ländern in Beziehung zueinander, zeigt sich, dass intergenerationale Leistungen bis auf wenige Ausnahmen weiter verbreitet und damit gesellschaftlich bedeutender sind als professionelle Unterstützungsangebote.

Sowohl hinsichtlich der Hilfe (Abbilvon informellen Pflegeleistungen erhalten sie von ihrem Partner oder ihrer Partnerin. Das ist allerdings nicht in allen Ländern der Fall. In den konservativen Wohlfahrtsstaaten Deutschland und Österreich sowie Italien werden mehr ältere Personen (auch) von den Kindern als von der Lebensgefährtin oder dem Lebensgefährten gepflegt.

Ein interessanter Unterschied zeigt sich bei den Personen im Unterstützungsnetzwerk: Während bei den definitionsgemäß weniger intensiven Hilfeleistungen auch Bekannte und Freunde eine wichtige Rolle spielen, sind es bei den intensiven und oft sehr intimen Pflegebeziehungen fast ausnahmslos die nächsten Angehörigen, die für die Pflege aufkommen.

Zusammenfassend kann gesagt werden, dass haushaltsübergreifende Hilfeund Pflegeleistungen vorwiegend von Kindern erbracht werden, wobei es deutliche Unterschiede zwischen den einzelnen Ländern gibt. Die Untersuchung der intergenerationalen Unterstützung ist demnach ein Schlüssel zur Erklärung der Unterschiede in der gesellschaftlichen Organisation von Hilfe und Pflege für ältere Menschen. Die Frage liegt also nahe, warum ältere Eltern in einem Land mehrheitlich von ihren Kindern unterstützt werden, Kinder in einem anderen Staat jedoch seltener Hilfe- und Pflegeleistungen erbringen. dung 8) als auch der Pflege (Abbildung 9) hat die Familie in den konservativen und familialistischen Wohlfahrtsstaaten Deutschland, Österreich und Italien eine weit größere Bedeutung als in den nordischen Wohlfahrtsstaaten Schweden und Dänemark. Es gibt jedoch auch feine Unterschiede: Intergenerationale Hilfe ist auch in den nordischen Wohlfahrtsstaaten und den Beneluxländern durchaus verbreitet, hinzu kommt eine gut ausgebaute professionelle Hilfe (Brandt/Szydlik 2008; Brandt 2009).

Pflege wird hingegen in den sozialdemokratischen Regimen mit Ausnahme Schwedens häufiger von professionellen Trägern geleistet (Rauch 2007; Sundström et al. 2006), wobei hier die geringe Anzahl an Pflegeempfängern (vor allem in Schweden) auch auf die fehlende Berücksichtigung der ausgeprägten stationären Pflege zurückzuführen ist. In Italien werden ältere Menschen fast ausschließlich von Angehörigen und eben auch den Kindern gepflegt. Professionelle Pflegedienste sind dagegen nur sehr eingeschränkt verfügbar (Haberkern/Szydlik 2008; Haberkern 2009).

\footnotetext{
3 Hilfeleistungen zwischen Partnern innerhalb des Haushaltes wurden nicht erhoben, weil sich hierbei kaum unterscheiden lässt, welche Tätigkeiten Hilfeleistungen für andere sind, und wer wem bei einer Tätigkeit im gemeinsamen Haushalt geholfen hat.
} 
Aufgrund der geringen Fertilität und der steigenden Frauenerwerbstätigkeit könnten Angehörige die Pflege in Zukunft möglicherweise nicht mehr im gleichen Umfang übernehmen. Wenn dieser Rückgang der familialen Pflegeleistungen nicht durch einen Ausbau der (öffentlich finanzierten) professionellen Pflegeleistungen aufgefangen wird, dann muss - wie das Beispiel Italien zeigt - mit einem raschen Wachstum des grauen Pflegemarktes gerechnet werden. Bereits heute greifen Familien in Italien häufiger auf illegale Pflegemigrantinnen aus Niedriglohnländern zurück als auf professionelle Pflegedienste (Da Roit 2007; Gori 2000).

\subsection{MULTIVARIATE ANALYSE}

Bisher wurde vor allem die gesellschaftliche Organisation der Hilfe und Pflege in den Blick genommen, genauer: die Unterschiede und Gemeinsamkeiten zwischen den Ländern. Die Entscheidung für ein bestimmtes Pflegearrangement, für oder gegen die informelle oder familiale Unterstützung erfolgt jedoch in der Familie. Welche Bedürfnis-, Opportunitäts-, Familien- und kulturell-kontextuellen Strukturen (Szydlik 2000; 2008) beeinflussen diese Entscheidung? Unter welchen Bedingungen erhalten Eltern Hilfe oder Pflege von ihren Kindern, und wann von sozial-medizinischen Fachkräften? Unter welchen Bedingungen sind ältere Menschen in Europa einem erhöhten Risiko der Unterversorgung an Hilfe- oder Pflegeleistungen ausgesetzt?

Tabelle 1 enthält die Ergebnisse multinomialer logistischer Regressionen (Hilfe links, Pflege rechts), wobei gezeigt wird, welche individuellen, familialen und kulturell-kontextuellen Faktoren sich auf die Hilfe- und Pflegesituation von Eltern auswirken, also auf die Wahrscheinlichkeit, dass sie von ihren Kindern oder von Fachkräften Unterstützung erhalten oder einer Unterversorgung mit sozial-medizinischen Leistungen ausgesetzt sind. Positive Werte geben dabei an, dass die Wahrscheinlichkeit eines bestimmten Hilfe- oder Pflegearrangements in Abhängigkeit von einer bestimmten Eigenschaft größer ist als keine Unterstützung zu erhalten, negative Werte bedeuten entsprechend eine geringere Wahrscheinlichkeit.

Mit höherem selbst eingeschätztem Bedarf steigt auch die Wahrscheinlichkeit, dass die Befragten tatsächlich Hilfe oder Pflege erhalten, wobei sich Bedürfnisse
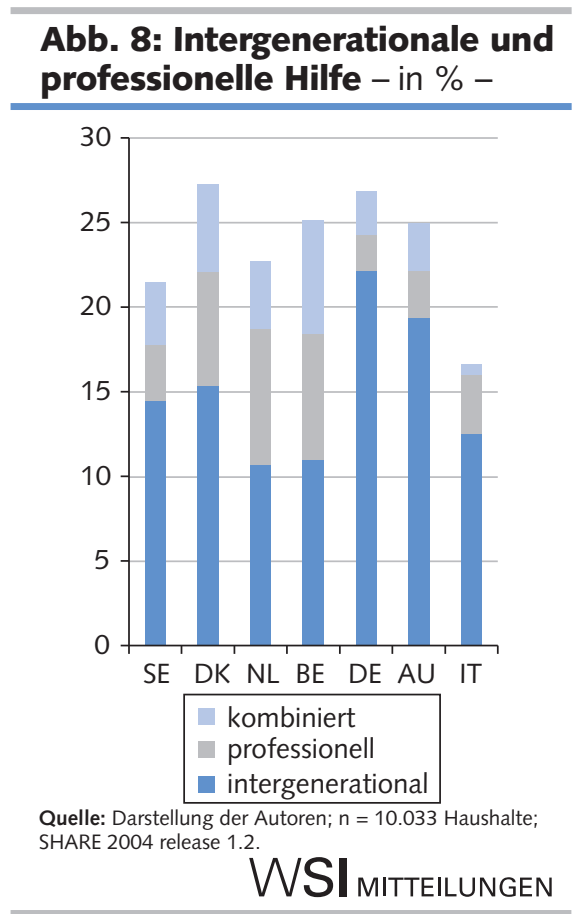

insbesondere auf eine kombinierte Unterstützung durch professionelle Dienstleister und Kinder auswirken. Bei ausgeprägtem Bedarf wird demnach eine professionelle Unterstützung eher zur intergenerationalen Unterstützung hinzugezogen. Das Alter der Befragten und ihre jährlichen Arztbesuche können ebenfalls als Indikatoren für die soziale und medizinische Notwendigkeit der Unterstützung aufgefasst werden. Mit steigendem Alter und mit der Anzahl der Arztbesuche erhalten Eltern eher Unterstützung durch Kinder oder professionelle Kräfte, wobei Arztbesuche nur einen geringen Effekt bei Pflege haben und sich dort insbesondere auf die Inanspruchnahme professioneller ambulanter Pflegeleistungen auswirken.

Ein gutes Haushaltsauskommen (subjektiv mindestens ausreichende finanzielle Ressourcen) begünstigt einerseits die Pflege durch Kinder, andererseits den Bezug professioneller Pflegeleistungen. Dies verdeutlicht, dass wohlhabende Personen ein geringeres Risiko der pflegerischen Unterversorgung tragen. Zudem ist es ein Hinweis darauf, dass die Entscheidung für oder gegen die Pflege der Eltern auch nach pragmatischen und strategischen $\mathrm{Ge}$ sichtspunkten erfolgen könnte. Zumindest engagieren sich Kinder eher in der Pflege der Eltern, wenn diese wohlhabend sind und die Kinder sich als "Gegenleistung“ für die Pflege Geldgaben erhoffen können. Eltern erhalten zudem eher Pflege von Kin-

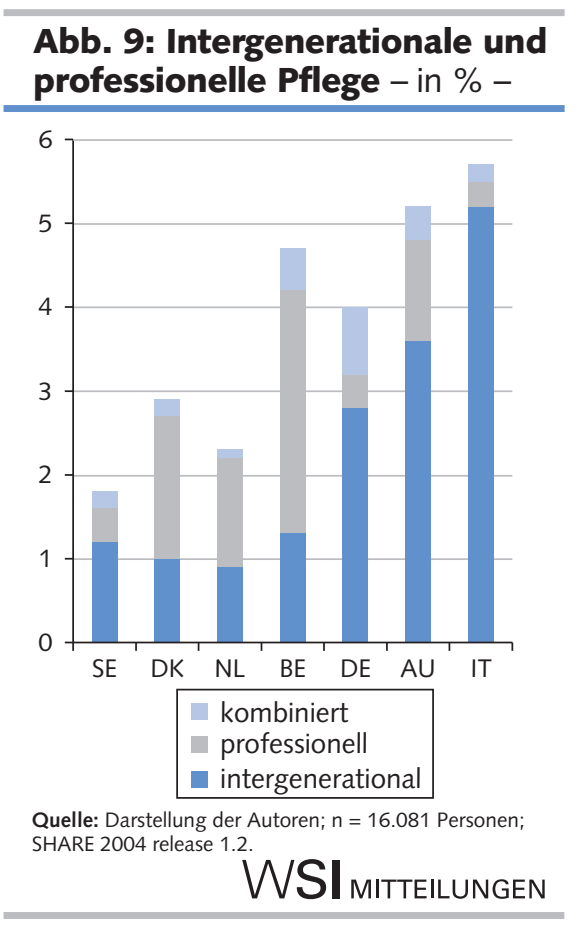

dern, wenn eine Erbschaft nicht ganz sicher ist. Die Pflege der Kinder könnte also dazu dienen, das Erbe zu erhalten und eher bei der Hinterlassenschaft bedacht zu werden. Zudem scheinen Personen mit höherer Bildung seltener Hilfe von ihren Kindern, jedoch eher von professionellen Dienstleistern in Anspruch zu nehmen, was einerseits mit einer stärkeren Autonomiebestrebung höher Gebildeter, aber auch mit verfügbaren Ressourcen zusammenhängen könnte (Theobald 2005).

Die Familienstrukturen wirken sich ebenfalls deutlich auf Unterstützungsarrangements aus: Eltern mit einem Partner erhalten seltener Pflege von Kindern oder von professionellen Pflegekräften, da in der Regel der Partner die Pflege übernimmt und zusätzliche Unterstützung meist erst sehr spät hinzugezogen wird. Männer tragen hierbei ein höheres Risiko, keine Pflege von Kindern oder Pflegediensten zu erhalten, sie werden jedoch häufiger von ihren Partnerinnen umsorgt. Alleinlebende Frauen und Männer erhalten ebenfalls häufiger Hilfe als zusammenlebende Paare. Besonders interessant ist der Einfluss der weiteren Zusammensetzung der Familie: Eltern mit mehreren Kindern erhalten eher Hilfe, egal, ob es sich um Töchter oder Söhne handelt. Bei der Pflege zeigt sich hingegen ein klares Geschlechtermuster: Sofern Eltern nur Töchter haben, werden sie eher gepflegt. Eltern, die nur Söhne haben, tragen hingegen ein höheres Risiko, 
Tabelle 1: Determinanten für den Erhalt von Hilfe und Pflege (Multinomiale logistische Regressionen)

\begin{tabular}{|c|c|c|c|c|c|c|}
\hline & \multicolumn{3}{|c|}{ Hilfearrangement } & \multicolumn{3}{|c|}{ Pflegearrangement } \\
\hline & Intergenerationales & Professionelles & Kombiniertes & Intergenerationales & Professionelles & Kombiniertes \\
\hline \multicolumn{7}{|c|}{$\begin{array}{l}\text { Opportunitäts- und Bedürfnisstrukturen } \\
\text { (Referenzkategorie: keine Unterstützung) }\end{array}$} \\
\hline Bedarf (IADL bzw. ADL) & $0.44^{* * *}$ & $0.81 * * *$ & $0.95^{* * *}$ & $0.59 * * *$ & $0.48^{* * *}$ & $0.82 * * *$ \\
\hline Alter & $0.03 * * *$ & $0.08 * * *$ & $0.11 * * *$ & 0.01 & $0.05^{* * *}$ & $0.06^{* *}$ \\
\hline Arztbesuche & $0.02 * * *$ & $0.02 * * *$ & $0.03 * * *$ & 0.01 & $0.02 * *$ & 0.01 \\
\hline Haushaltsauskommen gut & -0.04 & -0.19 & -0.12 & $0.51 *$ & $0.59^{*}$ & 0.21 \\
\hline Erbschaft unsicher & - & - & - & $1.06^{* *}$ & -0.76 & $1.13^{*}$ \\
\hline Bildung niedrig & & Referenz & & & Referenz & \\
\hline Bildung mittel & $-0.23 * *$ & $0.31^{*}$ & -0.14 & 0.01 & -0.02 & 0.14 \\
\hline Bildung hoch & $-0.30 * *$ & $0.38^{*}$ & -0.12 & -0.29 & -0.18 & -0.94 \\
\hline \multicolumn{7}{|l|}{ Familienstrukturen } \\
\hline Paar/Partner & & Referenz & & $-1.26^{* * *}$ & $-0.66 * *$ & $-1.43 * * *$ \\
\hline Mann & & & & $-0.83^{* *}$ & $-0.56^{*}$ & $-1.65^{* *}$ \\
\hline Frau allein & $1.11 * *$ & $0.89 * * *$ & $1.91 * * *$ & & & \\
\hline Nur Sohn & & Referenz & & & Referenz & \\
\hline Nur Tochter & -0.09 & 0.09 & -0.02 & $1.39 * * *$ & 0.40 & $1.66^{*}$ \\
\hline Tochter u. Sohn & $0.23 * *$ & -0.11 & 0.21 & $0.81 * *$ & 0.06 & $1.47^{*}$ \\
\hline \multicolumn{7}{|c|}{ Kulturell-kontextuelle Strukturen } \\
\hline Verantwortung Staat & 0.03 & $0.33^{* * *}$ & $0.23^{* *}$ & -0.13 & $0.26+$ & -0.07 \\
\hline Schweden & $-0.49 * * *$ & $0.53+$ & 0.38 & $-1.91 * *$ & 0.26 & -1.45 \\
\hline Dänemark & -0.19 & $1.55^{* * *}$ & $1.27 * * *$ & -1.01 & $1.53 * *$ & 0.57 \\
\hline Niederlande & $-0.71 * * *$ & $1.95 * * *$ & $1.10 * * *$ & -0.03 & $1.57 * * *$ & -0.58 \\
\hline Belgien & $-0.78^{* * *}$ & $1.54 * * *$ & $1.07 * * *$ & $-0.70+$ & $2.10 * * *$ & 0.57 \\
\hline Deutschland & & Referenz & & & Referenz & \\
\hline Österreich & $-0.23^{*}$ & 0.38 & 0.01 & $0.70^{*}$ & $0.98^{*}$ & 0.25 \\
\hline Italien & $-1.22 * * *$ & 0.00 & $-2.85 * * *$ & $1.15^{* * *}$ & -0.14 & 0.12 \\
\hline $\mathrm{n}$ & \multicolumn{3}{|c|}{9823 Haushalte } & \multicolumn{3}{|c|}{1.250 (pflegebedürftige) Personen } \\
\hline Pseudo $r^{2}$ & \multicolumn{3}{|c|}{0.19} & \multicolumn{3}{|c|}{0.25} \\
\hline
\end{tabular}

keine Pflege zu erhalten, auch wenn sie vergleichsweise häufig ambulante Pflegeleistungen beziehen (müssen). Insgesamt sprechen die Befunde dafür, dass Töchter eher selbst die Pflege übernehmen, während Söhne die Pflege den Schwestern oder professionellen Pflegediensten überlassen bzw. diese explizit beauftragen.

Nicht zuletzt beeinflussen kulturellkontextuelle Strukturen, ob und in welcher Form Unterstützungsleistungen erfolgen: Sehen die Befragten die Verantwortung für Hilfe für Angehörige eher beim Staat, nehmen sie auch eher professionelle oder kombinierte Hilfearrangements in Anspruch. Ebenso beziehen Eltern häufiger ambulante Pflegeleistungen, wenn sie die Pflegeverantwortung beim Staat und nicht in der Familie sehen. Auch unter Berücksichtigung dieser Einstellungen zeigen sich deutliche Länderunterschiede: Im Vergleich zu Deutschland erhalten ältere Eltern insbesondere in den Benelux-Ländern und den nordischen Wohlfahrtsstaaten eher Unterstützung von professionellen Anbietern, dagegen seltener von ihren
Kindern. In Deutschland und Österreich ist die familiale Unterstützung hingegen weit verbreitet. Pflege erfolgt in Italien vorwiegend in der Familie, Kinder leisten dort im Vergleich zu Deutschland seltener Hilfe. Nimmt man das geringe Niveau der professionellen Hilfe in Italien hinzu, dann ist dort das Risiko, im Alter keine Unterstützung zu erhalten, am höchsten, wobei dieses Risiko durch die Beschäftigung illegaler Pflegemigrantinnen („badanti“) reduziert wird (Da Roit 2007).

Intergenerationale Hilfe- und Pflegeleistungen stehen demnach im Zusammenhang mit unterschiedlichen Wohlfahrts- und Familienregimen (z. B. Leitner 2003). Je mehr Verantwortung der Staat für unterstützungsbedürftige Familien (-mitglieder) übernimmt, desto eher erfolgt die Unterstützung durch professionelle Anbieter. Eine Verdrängung (crowding out) familialer intergenerationaler Leistungen, wie sie hier untersucht wurden, zeigt sich vor allem bei der regelmäßigen Pflege; sporadische Hilfen werden in allen untersuchten Ländern vorrangig in der Familie geleistet.

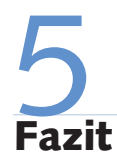

Die Unterstützung älterer Menschen ist im Zuge der demografischen Alterung ein aktuelles und vieldiskutiertes Thema. Im vorliegenden Beitrag wurde untersucht, wie die Unterstützung älterer Menschen in europäischen Ländern gesellschaftlich organisiert ist. Neben der Frage, ob hilfe- und pflegebedürftige Personen auch auf eine Unterstützung zählen können, wurde auch analysiert, wer welche Aufgaben übernimmt und welche Bedeutung informelle und formelle Unterstützungsnetzwerke in Ländern Nord-, Mittel- und Südeuropas haben. Schließlich wurde der Frage nachgegangen, ob und, wenn ja, welche individuellen und familialen Unterschiede in der Inanspruchnahme von intergenerationalen und professionellen Hilfe- und Pflegeleistungen bestehen.

Zusammenfassend lässt sich festhalten: Auf gesellschaftlicher Ebene steigen 
in den untersuchten Ländern mit dem Bedarf auch die Hilfe- und Pflegeleistungen an. Allerdings erhalten nicht alle pflegebedürftigen Personen die benötigte Unterstützung. Da in fast allen Pflegesystemen ein erheblicher oder schwerer Pflegebedarf nachgewiesen werden muss, bevor öffentliche oder Pflegeleistungen aus Versicherungen bezogen werden können, dürften vor allem Personen mit geringem oder mittelschweren Pflegebedarf keine Unterstützung erhalten und verstärkt von einer Unterversorgung betroffen sein, besonders wenn im persönlichen Umfeld keine Helfer zur Verfügung stehen. Insgesamt übersteigt der Pflegebedarf damit die Pflegeleistungen. Bei Hilfe finden sich hingegen keine Hinweise auf eine umfassende Unterversorgung. Sie wird häufig sogar auch dann geleistet, wenn keine explizite Notwendigkeit besteht. Dieses Ergebnis unterstreicht einmal mehr, dass unterschiedliche Unterstützungsformen auch separat betrachtet und analysiert werden müssen, wenn es um den Einfluss von individuellen, familialen und gesellschaftlichen Strukturen auf die Unterstützung älterer Menschen geht (Brandt et al. 2009).

Insgesamt tragen Kinder (und Partner) die Hauptlast der Unterstützung für Ältere, wobei es jedoch deutliche Unterschiede zwischen den Ländern gibt. So ist die informelle Pflege in dem familialistischen Wohlfahrtsstaat Italien und den konserva- tiven Wohlfahrtsstaaten Deutschland und Österreich sowohl relativ als auch absolut von größerer Bedeutung als formelle Pflegeleistungen - an weit über $80 \%$ der Pflegearrangements in Privathaushalten sind Angehörige und Bekannte aktiv beteiligt. In den nordischen Ländern, den Niederlanden und Belgien kommen professionelle Pflegedienste für einen höheren Anteil der Pflegeleistungen auf und sind sogar weiter verbreitet als die intergenerationale Pflege. Ein ähnliches Bild zeigt sich bei Hilfeleistungen. Angebot und Inanspruchnahme professioneller hauswirtschaftlicher Hilfen sind in den skandinavischen und den Beneluxländern höher; in Deutschland, Österreich und Italien erbringen Kinder und Angehörige hingegen klar das Gros der Hilfeleistungen. Neben diesen Unterschieden zwischen Ländern bestehen vor allem bei medizinisch anspruchsvollen, regelmäßig notwendigen Pflegeleistungen Ungleichheiten. Personen mit größeren finanziellen Ressourcen erhalten eher intergenerationale Pflege und können kostenintensive professionelle Unterstützung in Anspruch nehmen.

Auf der Basis der Analyse des Zusammenspiels professioneller und familialer Hilfe- und Pflegeleistungen lassen sich damit folgende Schlussfolgerungen ziehen: Während gegenwärtig der Hilfebedarf weitgehend gedeckt werden kann, weisen bereits mehrere Indikatoren auf Versor- gungslücken in der Pflege hin. Dies gilt in unterschiedlichem Ausmaß für alle hier untersuchten Länder, sodass von einer strukturellen Unterversorgung in Pflegesystemen gesprochen werden muss. Eine höhere Erwerbsbeteiligung jüngerer Frauen und Mütter sowie die geringen Fertilitätsraten könnten in Zukunft das familiale Hilfe- und Pflegepotenzial reduzieren, was die Versorgungslücke sogar vergrößern würde. Diese Entwicklung dürfte vor allem eine Herausforderung für Deutschland, Italien und Österreich darstellen, wo Hilfe- und Pflegeleistungen vorwiegend von Töchtern und Söhnen erbracht werden. Die skandinavischen Staaten sowie die Beneluxländer scheinen mit dem höheren Anteil an professionellen Serviceleistungen für Ältere dagegen besser auf die andauernden sozio-demografischen Veränderungen vorbereitet zu sein. Zudem entlasten die ausgebauten professionellen Dienste die Familie von zum Teil medizinisch anspruchsvollen Leistungen für ältere Angehörige und ermöglichen damit eher die Wahl zwischen verschiedenen Lebensentwürfen, die die Unterstützung von Angehörigen genauso einschließen können wie eine umfassende Erwerbstätigkeit. Doch auch hier gilt: Ohne die Unterstützung durch die Familie kann eine Gesellschaft den Bedürfnissen der älteren Generation zumindest gegenwärtig nicht gerecht werden.

\section{LITERATUR}

Attias-Donfut, C./Wolff, F.-C. (2000): Complementarity Between Private and Public Transfers, in: Arber, S./Attias-Donfut, C. (Hrsg.) The Myth of Generational Conflict: The Family and State in Ageing Societies, London, S. 47-68

Blinkert, B./Klie, T. (2004): Gesellschaftlicher Wandel und demografische Veränderungen als Herausforderungen für die Sicherstellung der Versorgung pflegebedürftiger Menschen, in: Sozialer Fortschritt 11/12, S. 319-325

\section{Bundesministerium für Familie, Senioren, Frauen und Jugend}

(BMFSFJ) (Hrsg.) (2006): Familie zwischen Flexibilität und Verlässlichkeit - Perspektiven für eine lebenslaufbezogene Familienpolitik, Siebter Familienbericht, Berlin

Börsch-Supan, A./Jürges, H. (Hrsg.) (2005): Health, Ageing and Retirement in Europe - Methodology, Mannheim
Brandt, M./Szydlik, M. (2008): Soziale Dienste und Hilfe zwischen Generationen in Europa, in: Zeitschrift für Soziologie 4, S. 301-320 Brandt, M. (2009): Hilfe zwischen Generationen. Ein europäischer Vergleich, Wiesbaden

Brandt, M./Haberkern, K./Szydlik, M. (2009), Intergenerational Help and Care in Europe, in: European Sociological Review 5, S. 585-601 Daatland, S. O./Herlofson, K. (2003): Families and Welfare States: Substitution or Complementarity, in: Lowenstein, A./Ogg, J. (Hrsg.): OASIS: Old Age and Autonomy - The Role of Service Systems and Intergenerational Family Solidarity. The Final Report, Haifa, S. 281-305

Da Roit, B. (2007): Changing International Solidarities Within Families in a Mediterranean Welfare State: Elderly Care in Italy, in: Current Sociology 2, S. 251-269 
Gori, C. (2000): Solidarity in Italy's Policies Towards the Frail Elderly: A Value at Stake, in: International Journal of Social Welfare 4, S. 261-269 Haberkern, K. (2009): Pflege in Europa - Familie und Wohlfahrtsstaat, Wiesbaden

Haberkern, K./Szydlik, M. (2008): Pflege der Eltern - ein europäischer Vergleich, in: Kölner Zeitschrift für Soziologie und Sozialpsychologie 1,

S. 78-101

Hank, K. /Buber, I. (2009): Grandparents Caring for Their Grandchildren: Findings From the 2004 Survey of Health, Ageing and Retirement, in: Journal of Family Issues 1, S. 53-73

Höpflinger, F./Hummel, C./Hugentobler, V. (2006): Enkelkinder und ihre Grosseltern. Intergenerationelle Beziehungen im Wandel, Zürich Igel, C./Brandt, M./Haberkern, K./Szydlik, M. (2009): Specialization Between Family and State. Intergenerational Time Transfers in Western Europe, in: Journal of Comparative Family Studies 2, S. 203-226

Leitner, S. (2003): Varieties of Familialism - The Caring Function of the Family in Comparative Perspective, in: European Societies 4, S. 353-375 Lowenstein, A./Ogg, J. (Hrsg.) (2003): OASIS: Old Age and Autonomy - The Role of Service Systems and Intergenerational Family Solidarity, The Final Report, Haifa

Motel-Klingebiel, A./Tesch-Römer, C. (2006): Familie im Wohlfahrtsstaat - Zwischen Verdrängung und gemischter Verantwortung, in:

Zeitschrift für Familienforschung 3, S. 290-314
Pfau-Effinger, B. (2005): Development Paths of Care Arrangements in the Framework of Family Values and Welfare Values, in: Pfau-Effinger, B./Geissler, B. (Hrsg): Care and Social Intergration in European Societies, Bristol, S. 21-45

Rauch, D. (2007): Is There Really a Scandinavian Social Service Model? in: Acta Sociologica 3, S. 249-269

Schneekloth, U./Leven, I. (2003): Hilfe- und Pflegebedürftige in Privathaushalten in Deutschland 2002 - Schnellbericht, München

Sundström, G./Malmberg, B./Johansson, L. (2006): Balancing Family and State Care: Neither, Either or Both? The Case of Sweden, in: Aging \& Society 5, S. 767-782

Szydlik, M. (2000): Lebenslange Solidarität? Generationenbeziehungen zwischen erwachsenen Kindern und Eltern, Opladen

Szydlik, M. (2008): Intergenerational Solidarity and Conflict, in: Journal of Comparative Family Studies 1, S. 97-114

Theobald, H. (2005): Social Exclusion and Care for the Elderly - Theoretical Concepts and Changing Realities in European Welfare States, WZB Discussion Paper SP I 301, Berlin

Walker, A./Pratt C./Eddy, L. (1995): Informal Caregiving to Aging Family Members: A Critical Review, in: Family Relations 4 (Helping Contemporary Families), S. 402-411 